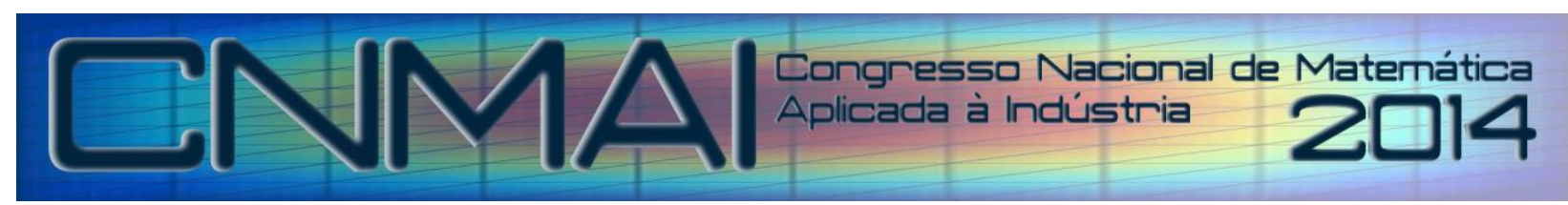

18 a 21 de novembro de 2014, Caldas Novas - Goiás

\title{
MULTIOBJECTIVE OPTIMIZATION OF COMPOSITE STRUCTURES WITH PARAMETRIC UNCERTAINTIES FOR ROBUST DESIGN
}

\author{
CNMAI2014-0046
}

\begin{abstract}
In the context of new developments in mechanical engineering, composite materials have been used in a large number of structures such as airplanes, compressors systems, automobiles, communalizations satellites, robotics, etc, due to their great flexibility in obtaining a great performance as a result of the combination of a number of parameters such number of layers with their thicknesses and the fiber orientations. However, in a design phase of a real-word composite structure of industrial interest, the question is how the arrangements of these parameters may affect the performance of such structures? Moreover, in the quest of design, another aspect that must be pointed out is the use of a robust optimization strategy in order to generate the best solutions with a reasonable robustness quantity. In this paper, the interest is confined to the finite element modeling procedure of composite structures by using the well-kwon higher-order shear deformation theory (HSDT). Emphasis is placed on a parameterization procedure in order to facilitate the introduction of the parametric uncertainties on the design parameters factored-out of the elementary matrices of the system. Furthermore, with the aim of generating optimal and robust design, the strategy used herein is to introduce vulnerability functions to be optimized as the same time as the original cost functions by using a multi-objective optimization procedure based on the Pareto construction. The probability distribution function for the uncertainty variables such as the thickness of the layers and the fiber orientations, was assumed based on the Maximum Entropy Principle, and the results in terms of the envelopes of the frequency response functions have been obtained and the robustness of the robust-optimal point has been verified a posteriori.
\end{abstract}

Keywords: Composite materials, robust optimization, vulnerability function, uncertainties.

\section{INTRODUCTION}

The last few decades has been the most significant for composite structures, which is gaining lot of space compared to the traditional materials used in engineering, such as metal alloys, with a wide range of applications in various areas such as aeronautics, aerospace, marine, automotive, bioengineering, construction, among others (Levy Neto, Pardini, 2006). As an example of the above in the previous paragraph can cite the aviation industry, in which major aircraft manufacturers are increasing more and more the use of these materials in their aircraft, like the Airbus A380, which has structural elements with important functions made of composite materials, or as the Boeing B787 that has most of its fuselage made of composite (Irisarri, 2009).

Other branches of industry that the composites are gaining strength are automotive and petroleum industries. In the automobile industry, composites are already used in the crankcase, bumpers, springs, panels, etc.. In the oil industry can highlight the use in risers.

This class of materials is very broad encompassing fiber reinforced polymers, structural concrete, metal matrix composites or ceramic, among others. Thus, the composites are characterized as combinations of two or more different materials at a macroscopic level, which in most cases are distinguished at least two phases called matrix and reinforcement (Levy Neto and Pardini, 2006; Souza, 2003).

The purpose of these materials, in addition to seeking the individual characteristics of each material component, is to obtain synergistic effects resulting from this combination, in other words, that no special properties of the 
constituents present individually as simultaneously display stiffness and low density, or mechanical strength and corrosion resistance (Hull, Clyne, 1975). Besides these very desirable features for designers, composites still exhibit other characteristics of great value:

- Mechanical properties can be "customized" according to the arrangement of fibers to meet the requirements of the project;

- Low sensitivity to fatigue and corrosion, reducing maintenance costs.

Due to the presented, it is found that the composites have a higher complexity when compared to more conventional isotropic materials since the properties of the composite are significantly influenced by a large number of variables and factors, in particular the variety of possible constructive forms. But if one side has a more complex mathematical model, on the other hand, has greater freedom constructively in the manufacture of these materials, which are suitable to meet the various project needs.

Exists in the literature a wide variety of theories that propose the modeling of laminar composite structures, which have different advantages and disadvantages, such as accuracy, especially regarding the shear effects along the thickness, computational cost, its main differences come from the polynomials order chosen to approximate the mechanical variables (Reddy, 1997). In this study will be used to Higher-order Shear Deformation Theory (HSDT).

With wide range of solutions is the use of an optimization algorithm is very useful for the designer, and as the vast majority of problems are multiobjectivos structural design, characterized by the presence of multiple objective functions which are often conflicting, optimal design is very important from the point of view of performance.

Among the various optimization algorithms, there are the evolutionary algorithms which are widely used in solving multiobjectives problems in different areas of engineering. In this work we will use a method of data analysis " $a$ posteriori".

In the design of complex structures, all steps require the use of reliable numerical models that can provide realistic qualitative and quantitative predictions of structural behavior. Such models are essential for the structural optimization and reliability assessment. With this goal, great research effort has been undertaken in recent years for the development of modeling techniques adapted to smart structures (Donadon, 2000)

The composite structures, as well as any system are subject to various uncertainties and errors at different levels, and more complex systems are even more subject, thus rely on an optimal design and / or robust is of great value for the requirements of reliability, safety, economy beyond the aforementioned performance. Here the uncertainties were applied to layer thickness and direction of the fibers, and the results analyzed using the envelopes of the frequency response.

\section{FINITE ELEMENT MODELING OF COMPOSITE PLATES}

This section will display the finite element formulation of a laminated composite plate shown in Fig. 1, where $\theta_{k}$, $z_{n}, h_{n}$ are, respectively, the orientation of the layers, the coordinate in the direction of plate thickness and width of each layers. It uses the Higher-Order Shear Deformation Theory developed by Lo et al., (1997); Chee et al. (2000), Lima et al. (2010), the displacement fields are shown in Eq. (1).

$$
\boldsymbol{U}(x, y, z, t)=\boldsymbol{A}(z) \widehat{\boldsymbol{u}}(x, y, t)
$$

where:

$$
\begin{aligned}
& \boldsymbol{U}(x, y, z, t)=\{\boldsymbol{u}(x, y, z, t) \boldsymbol{v}(x, y, z, t) \boldsymbol{w}(x, y, t)\}^{T} \\
& \boldsymbol{A}(z)=\left[\begin{array}{ccccccccccc}
1 & 0 & 0 & z & 0 & 0 & z^{2} & 0 & 0 & z^{3} & 0 \\
0 & 1 & 0 & 0 & z & 0 & 0 & z^{2} & 0 & 0 & z^{3} \\
0 & 0 & 1 & 0 & 0 & z & 0 & 0 & z^{2} & 0 & 0
\end{array}\right] \\
& \widehat{\boldsymbol{u}}(u)=\left\{\begin{array}{c}
u_{0}(x, y, t) v_{0}(x, y, t) w_{0}(x, y, t) \psi_{x}(x, y, t) \psi_{y}(x, y, t) \psi_{z}(x, y, t) \ldots \\
\ldots \zeta_{x}(x, y, t) \zeta_{y}(x, y, t) \zeta_{z}(x, y, t) \phi_{x}(x, y, t) \phi_{y}(x, y, t)
\end{array}\right\}
\end{aligned}
$$

where $\boldsymbol{u}(x, y, z, t), \boldsymbol{v}(x, y, z, t)$ and $\boldsymbol{w}(x, y, t)$ are the displacements in the directions $x, y$ and $z$, respectively. The terms $u_{0}, v_{0}$ and $w_{0}$ are, respectively, the mid-plane displacements of the plate in the directions $x, y$ and $z$, while $\psi_{x}, \psi_{y}$ and $\psi_{z}$ are the rotations around these axes. Already $\zeta_{x}, \zeta_{y}, \zeta_{z}, \phi_{x}$ and $\phi_{y}$ are high order rotations without defined physical interpretations (Chee et al., 2000). The vector shown in Eq (2-c) can be understood as the 11 variables or mechanical degrees of freedom used in this formulation. 


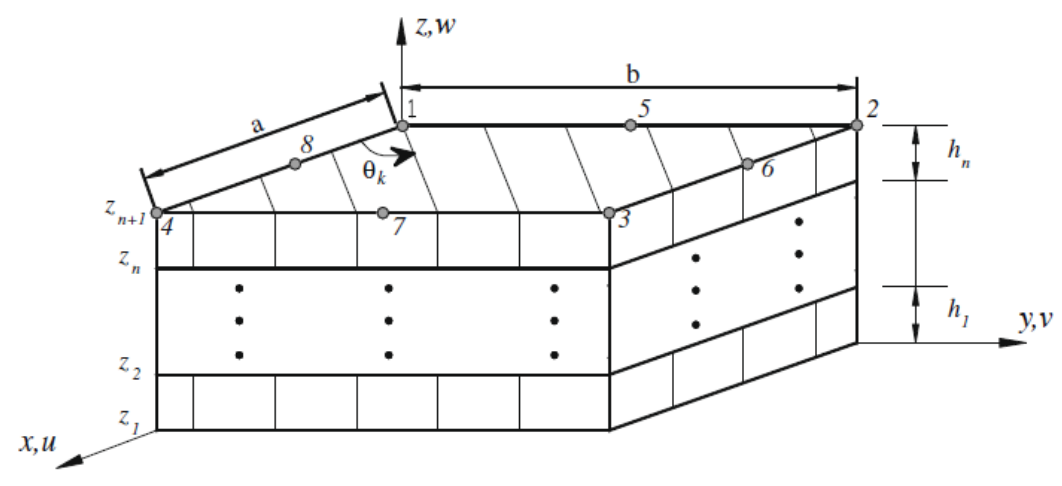

Figure 1. Laminated composite (adapted from de Lima et al. (2010))

Using the relationships coming from the theory of elasticity, the deformation fields can be found, and here they are divided into two groups: bending effects $\left(\boldsymbol{\varepsilon}_{\boldsymbol{b}}\right)$ and shear effects $\left(\boldsymbol{\varepsilon}_{\boldsymbol{s}}\right)$, as shown in Eq. (3).

$$
\begin{gathered}
\boldsymbol{\varepsilon}_{\boldsymbol{b}}=\left[\begin{array}{llll}
\boldsymbol{D}_{\mathbf{0}} & z \boldsymbol{D}_{\mathbf{1}} & z^{2} \boldsymbol{D}_{\mathbf{2}} & z^{3} \boldsymbol{D}_{3}
\end{array}\right] \widehat{\boldsymbol{u}} \\
\boldsymbol{\varepsilon}_{\boldsymbol{s}}=\left[\begin{array}{lll}
\boldsymbol{D}_{\mathbf{4}} & z \boldsymbol{D}_{\mathbf{5}} & z^{2} \boldsymbol{D}_{\mathbf{6}}
\end{array}\right]
\end{gathered}
$$

In Equations (3), $\boldsymbol{\varepsilon}_{\boldsymbol{b}}=\left\{\begin{array}{llll}\varepsilon_{x} & \varepsilon_{y} & \varepsilon_{z} & \gamma_{x y}\end{array}\right\}, \boldsymbol{\varepsilon}_{\boldsymbol{b}}=\left\{\begin{array}{ll}\gamma_{y z} & \gamma_{x z}\end{array}\right\}$ and the matrices $\boldsymbol{D}$ contains differential operators. Note that in Equation 3 the coordinate along the thickness $(z)$ was factored matrices of differential operators $\boldsymbol{D}$, which will be very important for the parameterization of the elementary matrices.

For a suitable finite element formulation it is necessary to describe the mechanical variables shown in Eq. (2-c) by appropriate choice of the finite element and shape functions to be used. In this formulation was chosen Serendip family element (Reddy, 1997; Faria, 2006), which is a plate element with eight nodes and eleven degrees of freedom per node, as shown in Fig. 2. With the shape functions, the 11 mechanical degrees of freedom can be related to the 88 nodal degrees of freedom as shown in Eq. (4).

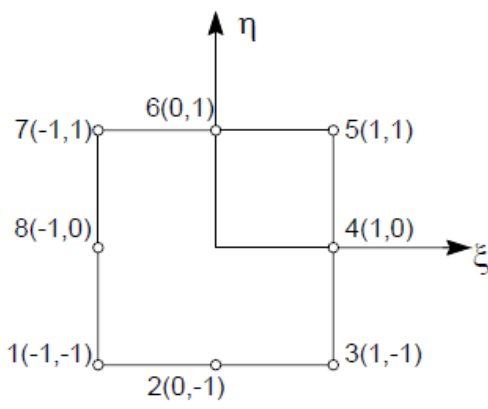

(A)

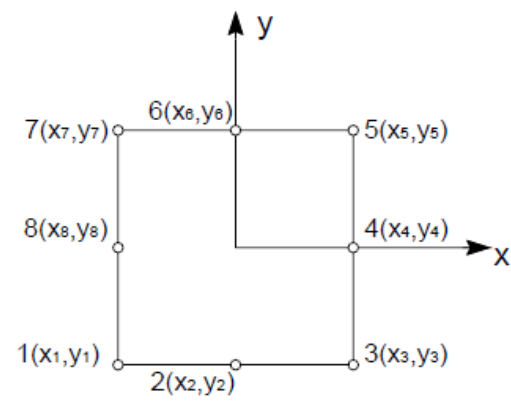

(B)

Figure 2. Rectangular element with eight nodes in local (A) and global (B) coordinates

$$
\widehat{\boldsymbol{u}}(\zeta, \eta, t)=\boldsymbol{N}(\zeta, \eta) \boldsymbol{u}_{e}
$$

Through the relationship shown in Eq. (4), the Eq. (1) and Eq. (3) may be rewritten as the equation presented in the Eq. (5).

$$
\begin{gathered}
\boldsymbol{U}(\zeta, \eta, z, t)=A(z) \boldsymbol{N}(\zeta, \eta) \boldsymbol{u}_{e} \\
\boldsymbol{\varepsilon}_{\boldsymbol{b}}=\left[\boldsymbol{D}_{\boldsymbol{b}} \boldsymbol{N}_{\boldsymbol{b}}\right] \boldsymbol{u}_{\boldsymbol{e}}=\boldsymbol{B}_{b} \boldsymbol{u}_{e} \\
\boldsymbol{\varepsilon}_{\boldsymbol{s}}=\left[\boldsymbol{D}_{\boldsymbol{s}} \boldsymbol{N}_{s}\right] \boldsymbol{u}_{e}=\boldsymbol{B}_{s} \boldsymbol{u}_{e}
\end{gathered}
$$

Using the interpolation of the displacement and deformation fields is possible to formulate the kinetic and potential energies that are respectively shown in Eq. (6) and Eq. (7).

$$
\boldsymbol{K}_{\boldsymbol{E}}(t)=\frac{1}{2} \dot{\boldsymbol{e}}_{\boldsymbol{e}}(t)^{T} \boldsymbol{M}_{\boldsymbol{e}} \dot{\boldsymbol{u}}_{\boldsymbol{e}}(t)
$$




$$
\boldsymbol{P}_{\boldsymbol{E}}(t)=\frac{1}{2} \boldsymbol{u}_{\boldsymbol{e}}(t)^{T} \boldsymbol{K}_{\boldsymbol{e}} \boldsymbol{u}(t)
$$

where:

$$
\boldsymbol{M}_{\boldsymbol{e}}=\sum_{k=1}^{n} \int_{z=z_{k}}^{z_{k=+1}} \int_{\zeta=-1}^{\zeta=+1} \int_{\eta=-1}^{\eta=+1} \rho_{k} \boldsymbol{A}(z) \boldsymbol{N}_{\boldsymbol{u}}(\zeta, \eta)^{T} \operatorname{det}(J) d \eta d \zeta d z
$$

is the matrix of elementary mass with $\rho_{k}$ being the density of the blade, considered homogeneous and $\boldsymbol{K}_{\boldsymbol{e}}=\boldsymbol{K}_{\boldsymbol{e}(\boldsymbol{b})}+$ $\boldsymbol{K}_{\boldsymbol{e}(\boldsymbol{s})}$ is the elementary stiffness matrix, which is formed by bending components, $\boldsymbol{K}_{\boldsymbol{e}(\boldsymbol{b})}$, and shear, $\boldsymbol{K}_{\boldsymbol{e}(\boldsymbol{s})}$, are expressed as:

$$
\begin{aligned}
& \boldsymbol{K}_{\boldsymbol{e}(\boldsymbol{b})}=\sum_{k=+1}^{n} \int_{k=+1}^{z_{k+1}} \int_{\zeta=-1}^{\zeta=+1} \int_{\eta=-1}^{\eta=+1} \boldsymbol{B}_{\boldsymbol{b}}(\zeta, \eta, z)^{T} \boldsymbol{C}_{\boldsymbol{b}}^{(\boldsymbol{k})}\left(\theta_{k}\right) \boldsymbol{B}_{\boldsymbol{b}}(\zeta, \eta, z) \operatorname{det}(J) d \eta d \zeta d z \\
& \boldsymbol{K}_{\boldsymbol{e}(\boldsymbol{s})}=\sum_{k=+1}^{n} \int_{k=+1}^{z_{k+1}} \int_{\zeta=-1}^{\zeta=+1} \int_{\eta=-1}^{\eta=+1} \boldsymbol{B}_{s}(\zeta, \eta, z)^{T} \boldsymbol{C}_{\boldsymbol{s}}^{(k)}\left(\theta_{k}\right) \boldsymbol{B}_{\boldsymbol{s}}(\zeta, \eta, z) \operatorname{det}(J) d \eta d \zeta d z
\end{aligned}
$$

In Eq. (8) matrices $\boldsymbol{C}_{\boldsymbol{b}}^{(\boldsymbol{k})}\left(\theta_{k}\right)$ and $\boldsymbol{C}_{\boldsymbol{s}}^{(\boldsymbol{k})}\left(\theta_{k}\right)$ are, respectively, the orthotropic elastic matrices associated with the bending and shear of each layer, which according to theory CLT are given by:

$$
\begin{gathered}
\boldsymbol{C}_{\boldsymbol{b}}^{(\boldsymbol{k})}\left(\theta_{k}\right)=\boldsymbol{T}_{\boldsymbol{b}}\left(\theta_{k}\right) \overline{\boldsymbol{C}}_{\boldsymbol{b}}^{(\boldsymbol{k})} \boldsymbol{T}_{\boldsymbol{b}}{ }^{\boldsymbol{T}}\left(\theta_{k}\right) \\
\boldsymbol{C}_{\boldsymbol{s}}^{(\boldsymbol{k})}\left(\theta_{k}\right)=\boldsymbol{T}_{\boldsymbol{s}}\left(\theta_{k}\right) \overline{\boldsymbol{C}}_{\boldsymbol{s}}^{(\boldsymbol{k})} \boldsymbol{T}_{\boldsymbol{s}}{ }^{\boldsymbol{T}}\left(\theta_{k}\right)
\end{gathered}
$$

where $\overline{\boldsymbol{C}}_{\boldsymbol{b}}^{(\boldsymbol{k})}$ and $\overline{\boldsymbol{C}}_{\boldsymbol{s}}^{(\boldsymbol{k})}$ are, respectively, the mechanical stiffness matrix containing the elastic properties of bending and shearing the k-th layer associated with the main orthotropic axis, and $\boldsymbol{T}_{\boldsymbol{b}}\left(\theta_{k}\right)$ and $\boldsymbol{T}_{\boldsymbol{s}}\left(\theta_{k}\right)$ are the associated rotation matrices. Using elementary matrices is possible to build the global equations through the Boolean connectivity matrix, which after assembly, the global equation of motion is given by:

$$
\boldsymbol{M} \ddot{\boldsymbol{q}}(t)+\boldsymbol{K q}(t)=\boldsymbol{f}(t)
$$

where $\boldsymbol{q}(t)$ is the vector containing the global degrees of freedom and $\boldsymbol{f}(t)$ is the vector containing the external loads.

\section{PARAMETERIZATION OF THE ELEMENTARY MATRICES}

For the better handling and a future sensitivities study, orientations of each layer were factored from the mechanical stiffness matrices, this procedure will be shown in this section. For layers of orthotropic material, the stiffness matrix for bending and shear, respectively, are given by:

$$
\begin{gathered}
\overline{\boldsymbol{C}_{\boldsymbol{b}}}=\left[\begin{array}{cccc}
C_{11} & C_{12} & C_{13} & 0 \\
C_{12} & C_{11} & C_{23} & 0 \\
C_{13} & C_{23} & C_{33} & 0 \\
0 & 0 & 0 & C_{66}
\end{array}\right] \\
\overline{\boldsymbol{C}_{\boldsymbol{s}}}=\left[\begin{array}{cc}
C_{44} & 0 \\
0 & C_{55}
\end{array}\right]
\end{gathered}
$$

For a global coordinate system, it is necessary to use a rotation operation around the axis $z$, as shown in Eq. (12).

$$
\begin{gathered}
\overline{C_{b}}=T_{b} C_{b} T_{b}{ }^{T} \\
\overline{C_{s}}=T_{s} C_{s} T_{s}{ }^{T}
\end{gathered}
$$

Matrices $\boldsymbol{T}_{\boldsymbol{b}}$ and $\boldsymbol{T}_{\boldsymbol{s}}$ are the transformation matrices in which the orientation of the layers may be factored as follows:

$$
\boldsymbol{T}_{\boldsymbol{b}}=\left[\begin{array}{cccc}
c_{k}^{2} & 1-c_{k}^{2} & 0 & -2 s_{k} c_{k} \\
1-c_{k}^{2} & c_{k}^{2} & 0 & 2 s_{k} c_{k} \\
0 & 0 & 1 & 0 \\
s_{k} c_{k} & -s_{k} c_{k} & 0 & 2 c_{k}^{2}-1
\end{array}\right]
$$




$$
\boldsymbol{T}_{\boldsymbol{s}}=\left[\begin{array}{cc}
c_{k} & S_{k} \\
-S_{k} & c_{k}
\end{array}\right]
$$

Where $c=\cos \theta$ and $s=\sin \theta$, and remembering that $s^{2}=1-c^{2}$

\section{ROBUST MULTI-OBJECTIVE OPTIMAZATION STRATEGY}

Recalling that the properties of each layer have a great influence on the properties of the composite structure, the design of these structures becomes very difficult because the large variety of possibilities, and are often complex to obtain the most desirable characteristics of these structures.

Having a robust optimal design is of great value to help the designer in choosing the most suitable design, considering the performance, cost and safety.

The vast majority of engineering problems have several objectives, often conflicting, and dependent on many variables. Thus, these multiobjetivos problems, unlike the mono-objective having more than one solution, being composed of a set of solutions known as Pareto solution.

A multi-objective problem can be defined as (Ait Brik, 2005)

$$
\left\{\begin{array}{c}
\min _{x} \boldsymbol{F}(\boldsymbol{x})=\left(f_{1}(\boldsymbol{x}), f_{2}(\boldsymbol{x}), \cdots, f_{n}(\boldsymbol{x})\right) \\
\boldsymbol{g}_{\boldsymbol{j}}(\boldsymbol{x}) \leq 0 j-1, \cdots, m \\
\boldsymbol{x}_{\boldsymbol{L}} \leq \boldsymbol{x} \leq \boldsymbol{x}_{\boldsymbol{U}} \boldsymbol{x} \in \boldsymbol{C}
\end{array}\right.
$$

where $n \geq 2$ is the number of objective functions, $\boldsymbol{x}=\left(x_{1}, x_{2}, \cdots, x_{k}\right)$ is the vector containing the design variables, $\boldsymbol{C} \subset \boldsymbol{R}^{\boldsymbol{k}}$ represents the feasible set (design space) associated with the equality constraints or inequality $\boldsymbol{g}_{\boldsymbol{j}}(\boldsymbol{x})$ and the explicit limits, $\boldsymbol{F}(x)$ is the vector of criteria or objective functions to be optimized.

There are several ways to obtain the set of solutions to the multi-objective problem as the notion of Nash's Equilibrium, or Pareto sense of equilibrium which is a principle commonly used in this type of problems. Pareto's equilibrium was defined by Wilfred Pareto and according to him, given a multi-objective problem, there is an equilibrium, such that it cannot be improved a criterion without degrading at least one other criterion.

The Pareto equilibrium is directly related to the notion of Pareto dominance, which can be defined as: a solution of $\boldsymbol{Y}=\left(y_{1}, \cdots, y_{n}\right)$ dominates another solution $\boldsymbol{Z}=\left(z_{1}, \cdots, z_{n}\right)$ if, and only if $\forall i \in[1, n], y_{i} \leq z_{i}$ and $\exists i \in[1, n]$ such that $y_{i}<z_{i}$. Then, a solution $x^{*} \in C$ is a Pareto solution if and only if there is no solution $\boldsymbol{x}^{*} \in C$ such that $\boldsymbol{F}(\boldsymbol{x})$ dominate $\boldsymbol{F}\left(\boldsymbol{x}^{*}\right)$ (Ait Brik, 2005).

Every real system is subject to the impact of various types of uncertainties to which it is exposed and right here is the robust optimization differs from deterministic optimization because the former takes into account the influence of some of these uncertainties.

In engineering, the uncertainties appear in all phases of design, fabrication, installation and operation, being inherent in any process, acting on the parameters, as well as the objective functions and constraints. The robust optimization aims to take into account some of these uncertainties in the project in order to take into account the lack of information about some design parameters, thus a robust optimal design is interesting to get a result safest and most economical without excessively degrading their performance.

Uncertainties impact of various forms in an optimization problem, as can be seen in Fig. (3) for a cost function of one variable, in which one sees the influence of the uncertainties on the design variables and other parameters that impact indirectly the calculation of the cost function.

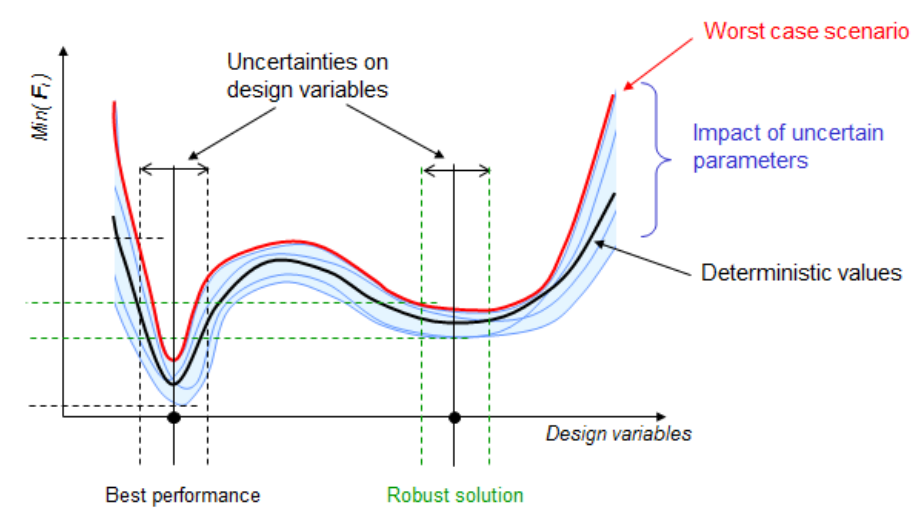

Figure 3. Impact of uncertainties in the cost function (extracted from Irisarri, 2009). 
To evaluate the impact of design variations is used here an estimator called robustness function that is based on the mean $(\mu)$ and standard deviation $(\sigma)$ of the cost functions and following the approach proposed by Ait Brik (2005), for an objective function $f(x)$, the robustness function is defined as:

$$
\boldsymbol{f}^{v}=\left(\sigma_{f} / \mu_{f}\right)^{-1}
$$

where $\left(\sigma_{f} / \mu_{f}\right)$ is a measure of the dispersion or vulnerability $f(x)$, denoted by $f^{v}(x)$.

So is defined a new robust multi-objective optimization problem, which simultaneously optimizes initial cost functions and robustness functions, and the problem with mathematically defined follows:

$$
\left\{\begin{array}{c}
\min _{x} F^{*}(\boldsymbol{x})=\left(f_{1}(\boldsymbol{x}), f_{1}^{v}(\boldsymbol{x}), f_{2}(\boldsymbol{x}), f_{2}^{v}(x), \cdots, f_{n}(\boldsymbol{x}), f_{n}^{v}(\boldsymbol{x})\right) \\
\boldsymbol{g}_{\boldsymbol{j}}(\boldsymbol{x}) \leq 0 j-1, \cdots, m \\
\boldsymbol{x}_{\boldsymbol{L}} \leq \boldsymbol{x} \leq \boldsymbol{x}_{\boldsymbol{U}} \boldsymbol{x} \in \boldsymbol{C}
\end{array}\right.
$$

\section{NUMERICAL SIMULATIONS}

Was implemented finite element model of a square plate of side $a=0,16 m$, as shown in Figure 4-A, the composite has five layers having the same elastic properties elastic properties: $\bar{E}_{1}=172,5 G P a ; \quad \bar{E}_{2}=\bar{E}_{3}=6,89 G P a$; $\bar{G}_{12}=\bar{G}_{13}=3,45 G P a ; v_{12}=v_{13}=0,25 ; v_{23}=0,30$ and density $\rho=1566 \mathrm{Kg} / \mathrm{m}^{3}$ is adopted for all layers.

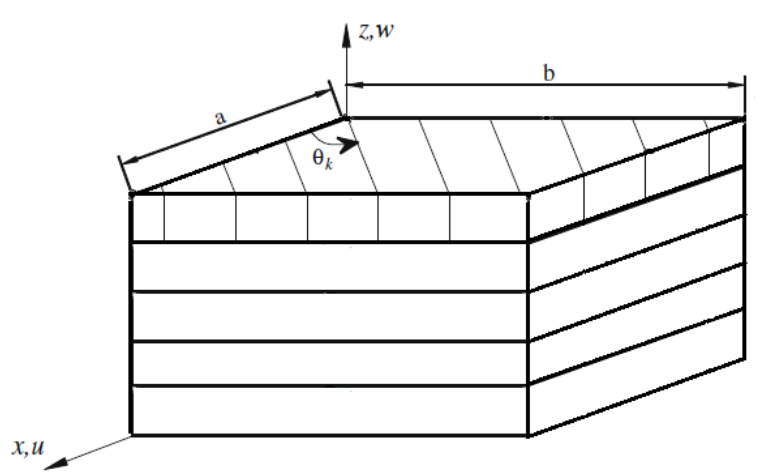

(A)

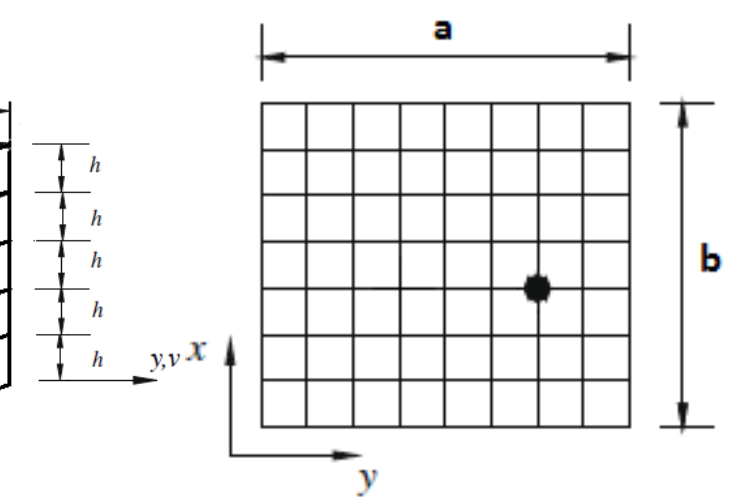

(B)

Figure 4. Geometry of the composite plate(A), mesh used (B)

We used a mesh with 64 finite elements, 225 nodes and the boundary conditions were used on the plate (Chee et al., 2000 ): $u_{0}=w_{0}=\psi_{x}=\zeta_{x}=\zeta_{z}=0$ with $y=0$ and $\mathrm{y}=\mathrm{b} ; u_{0}=w_{0}=\psi_{x}=\zeta_{y}=\zeta_{z}=0$ with $x=0$ and $x=a$.

The analysis presented here consist mostly FRFs at the point shown in Fig 4-B, with an aim of reducing the amplitudes of the first two frequency peaks. There were two optimizations, a robust and deterministic, in that the chosen nominal values are shown in Tab. 1. We used a variant of evolutionary algorithm called NSGA (Non Dominated Sorting Genetic Algorithm), the other settings used for optimization are: a population of 30 individuals in each generation, 100 generations with a mutation probability of 0,05 ; and 025 for the probability of crossing, for both the deterministic optimization and for robust.

After the end of the optimizations, was generating a point cloud containing a history of the evolution of solutions, which can be seen in Fig. 5-A, in Figure 5-B is the Pareto Front, is highlighted in red in the project nominal (initial), both for deterministic optimization.

Fig. 4-A shows a good performance of the algorithm solutions, converging to the Pareto front shown in Fig. 5-A, which is possible to realize solutions that are better performing than the nominal design. Point A shown in the figure was chosen to compare the amplitudes of the first two modes, as shown in Fig. 6, where for the optimal design was reduced by about $5 \mathrm{~dB}$ and $6 \mathrm{~dB}$, for the first and second peak, respectively. 
Table 1. Nominal values, deterministic and robust optimization.

\begin{tabular}{|l|l|c|c|}
\hline Design variables & nominal values & $\begin{array}{c}\text { permissible } \\
\text { variations }\end{array}$ & $\begin{array}{c}\text { levels of dispersion } \\
\text { in the design variables } \\
\text { (robust case) }\end{array}$ \\
\hline$h_{1}$ & $0,1 \times 10^{-3} \mathrm{~m}$ & $\pm 30 \%$ & $\Delta h=6 \%$ \\
\hline$h_{2}$ & $0,1 \times 10^{-3} \mathrm{~m}$ & $\pm 30 \%$ & $\Delta h=6 \%$ \\
\hline$h_{3}$ & $0,1 \times 10^{-3} \mathrm{~m}$ & $\pm 30 \%$ & $\Delta h=6 \%$ \\
\hline$h_{4}$ & $0,1 \times 10^{-3} \mathrm{~m}$ & $\pm 30 \%$ & $\Delta h=6 \%$ \\
\hline$h_{5}$ & $0,1 \times 10^{-3} \mathrm{~m}$ & $\pm 30 \%$ & $\Delta h=6 \%$ \\
\hline$\theta_{1}$ & $45^{\circ}$ & $\pm 4.5^{\circ}$ & $\Delta h=6 \%$ \\
\hline$\theta_{2}$ & $0^{\circ}$ & $\pm 4.5^{\circ}$ & $\Delta h=6 \%$ \\
\hline$\theta_{3}$ & $45^{\circ}$ & $\pm 4.5^{\circ}$ & $\Delta h=6 \%$ \\
\hline$\theta_{4}$ & $0^{\circ}$ & $\pm 4.5^{\circ}$ & $\Delta h=6 \%$ \\
\hline$\theta_{5}$ & $45^{\circ}$ & $\pm 4.5^{\circ}$ & $\Delta h=6 \%$ \\
\hline
\end{tabular}

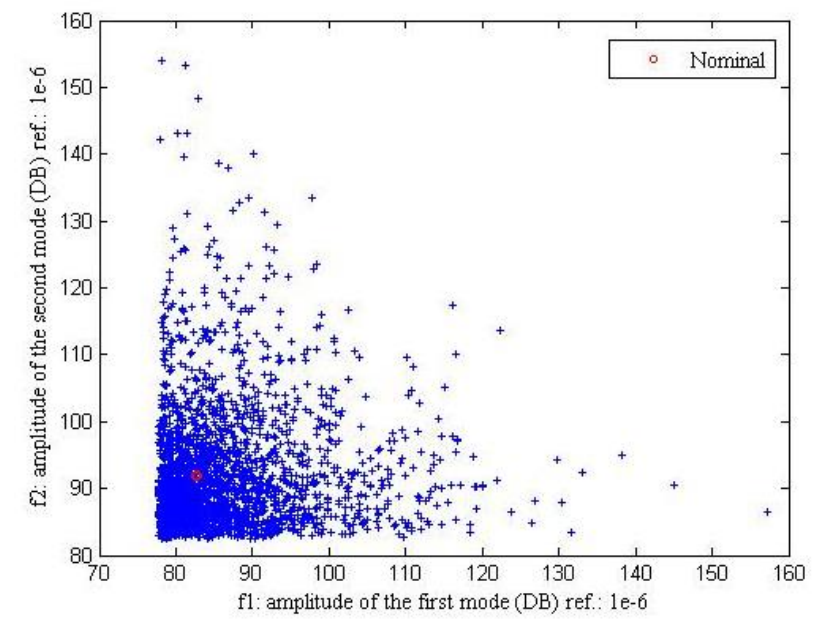

(A)

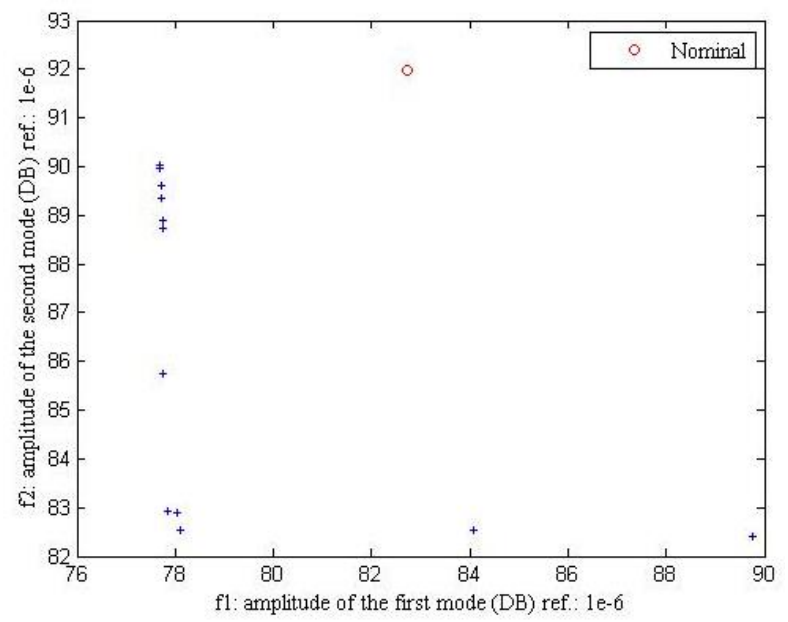

(B)

Figure 5. History solutions (deterministic optimization) (A); Pareto Front (B).

It was also generates a points cloud containing a history of robust solutions, as can be seen in Fig. 7. In Fig. 8 and Fig. 9 is shown a comparison between the FRF envelopes of deterministic and robust optimal designs, respectively, these envelopes were generated with $3 \%$ perturbation parameters through 50 samples using the Monte Carlo method. The analysis of the envelopes is possible to verify that the robust design (Fig. 9) is less subject to disruptions that the deterministic design (Fig. 8), with regard to the uncertainties introduced, which shows that the optimal solutions are very robust (minimum vulnerability) taking into account small perturbations, although not present the same level of reduction of frequency peaks that project deterministic. 


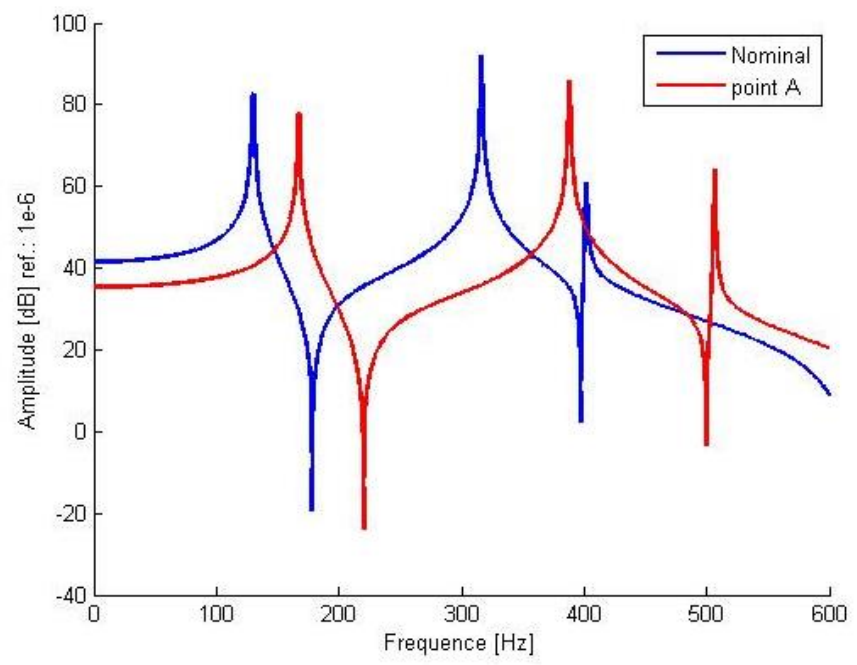

Figure 6. FRF comparison of the nominal design with A design

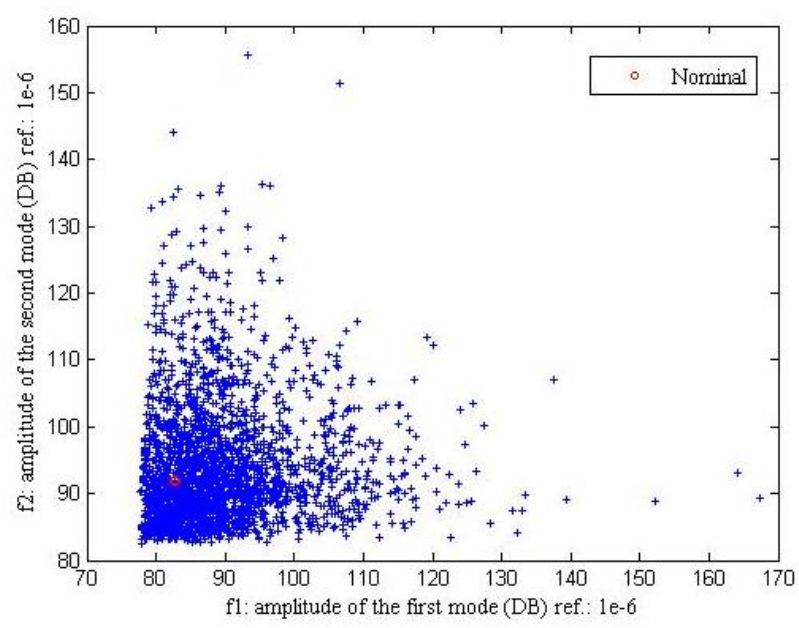

Figure 7. History solutions (robust optimization)

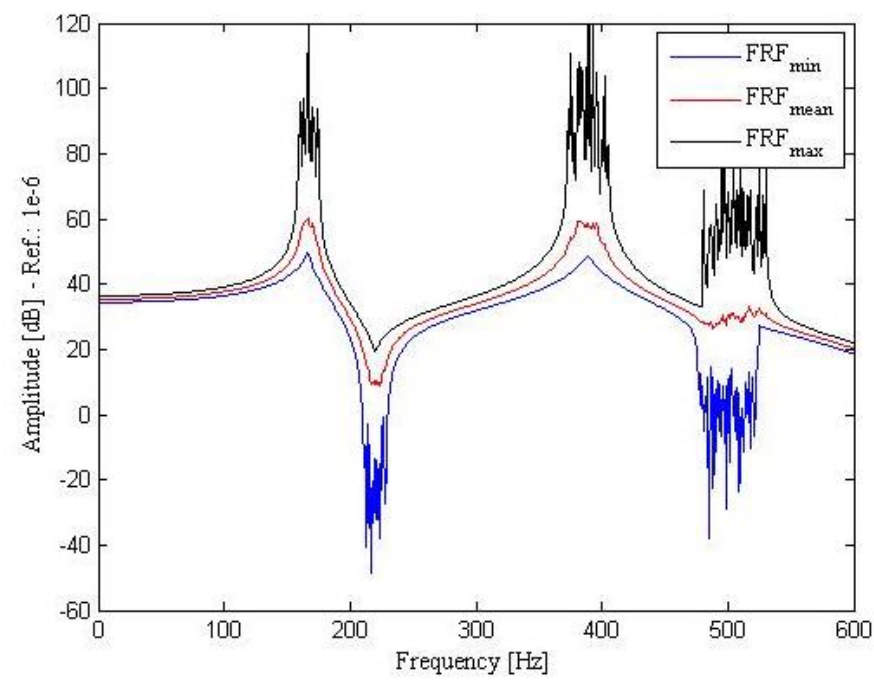

Figure 8. Envelope of deterministic design. 


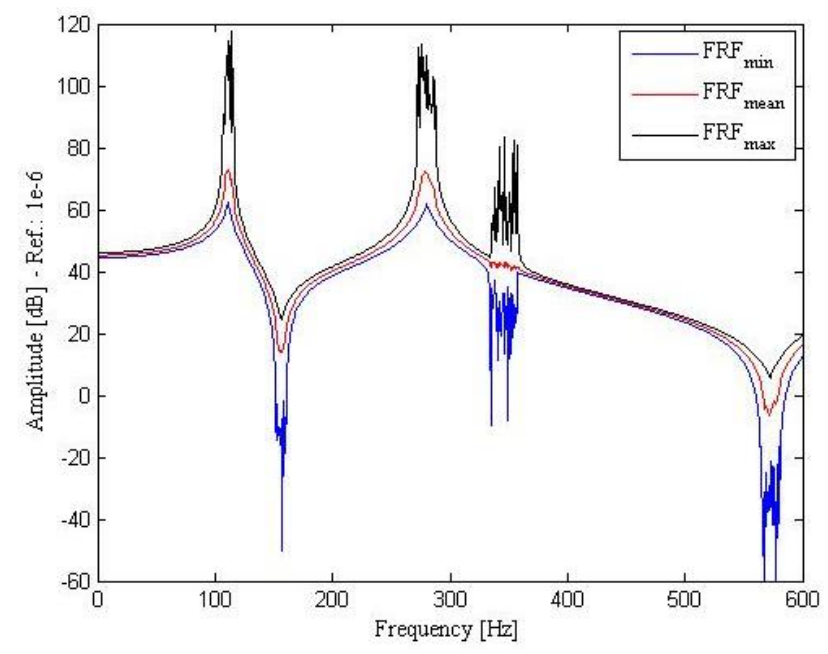

Figure 9. Envelope of robust design.

\section{CONCLUSIONS}

We implemented two multi-objective optimization routines using the algorithm NSGA, one deterministic and one robust, applied to a model of composite material that followed the theory HSDT where the thickness and orientation of the layers were the design variables, which were previously parameterized.

The parameterization of the model proved a good tactic to reduce the computational cost, especially to solve the integrations, improving the cost-benefit of theory HSDT, which has higher computational cost. The parameterization process also assisted in the manipulation of the model to study the robustness of the system, leaving a good possibility of a future sensitivity analysis.

A comparison of deterministic and robust optimizations, performed through the envelopes of FRFs demonstrated that as expected the robust design was less subject to variations caused by the oscillation introduced into the parameters, achieved by minimizing of the vulnerability functions.

Ignoring the problem of uncertainty is a mistake, because in one way or another are inevitable in real problems. The relevant question is not whether it should be taken into account, as an obligation, but to decide whether the desired costs and analysis are acceptable for its consideration.

\section{ACKNOWLEDGEMENTS}

The authors are thankful to the Brazilian Research Agencies CAPES Foundation, from the Brazilian Ministry of Education, for the financial support to this work through the INCT-EIE.

\section{REFERENCES}

Ait Brik B., 2005. Méthodologies de conception robuste et d'optimisation dans un contexte de conception d'architectures mécaniques nouvelles en avant projet. $\mathrm{PhD}$ Thesis, University of Franche-Comté, France.

Chee C, Tong L, Steven G., 2000. A mixed model for adaptive composite plates with piezoelectric for anisotropic actuation. Comput Struct; 77(3):253-68.

Donadon, M. V., 2000. Vibração de Placas Laminadas na Presença de Tensões Induzidas Piezoeletricamente. 109p. Master Thesis (Mechanical Engineering)- Technological Institute of Aeronautics, São José dos Campos, SP, Brazil.

Faria, A. W., 2006. Modelagem por elementos finitos de placas compostas dotadas de sensores e atuadores piezoelétricos: implementação computacional e avaliação numérica. 152p. Master Thesis (Mechanical Engineering). Faculty of Mechanical Engineering, Federal University of Uberlândia, MG, Brazil.

Hull, D.; Clyne, T. W., 1975. An Introduction to Composite Material. Scripta Book, Washigton.. $1^{\text {nd }}$ edition.

Irisarri, F. X., 2009. Statégies de calcul pour L'optimisation multiobjectif des structures composites. 274p. Ph.D. thesis, Universite de Toulouse III - Paul Sabatier, Toulouse, France.

Levy Neto, F.; Pardini, L. C., 2006. Compósitos Estruturais: Ciência e Tecnologia. Edgar Blücher, São Paulo. $1{ }^{\text {nd }}$ edition.

Lima, A. M. G.; Faria, A. W.; Rade, D. A., 2010. Sensitivity analysis of response functions of composite sandwich plates containing viscoelastic layers. Journal of Composite Structures. v. 92, n. 364-376.

Lo KH, Christensen RM, Wu EM., 1997. "A high order theory of plate deformation - part 2: laminated plates". J Appl Mech; 64(3):669-76.

Reddy, J. N., 1997. Mechanics of Laminated Composite Plates: Theory and Analysis. CRC Press, Florida. $2^{\text {nd }}$ edition. 
Congresso Nacional de Matemática Aplicada à Indústria, 18 a 21 de novembro de 2014, Caldas Novas - GO

Souza, C. E., 2003. Otimização em Dois Níveis da Orientação e da Topologia de Cascas Laminadas. 117p. Master Thesis - Federal University of Rio Grande do Sul, Porto Alegre, RS, Brazil.

\section{RESPONSIBILITY NOTICE}

"The authors are the only responsible for the printed material included in this paper". 